\title{
Creation, manipulation, and detection of Abelian and non-Abelian anyons in optical lattices
}

\author{
M. Aguado ${ }^{1}$, G. K. Brennen ${ }^{2}$, F. Verstraete ${ }^{3}$, J. I. Cirac ${ }^{1}$ \\ 1 Max-Planck-Institut für Quantenoptik, Hans-Kopfermann-Str. 1, Garching, D-85748, Germany \\ 2 Centre for Quantum Information Science and Security, Macquarie University, 2109, NSW Australia \\ 3 Fakultät für Physik, Universität Wien, Boltzmanngasse 5, A-1090 Wien, Austria.
}

(Dated: November 5, 2018)

\begin{abstract}
Anyons are particle-like excitations of strongly correlated phases of matter with fractional statistics, characterized by nontrivial changes in the wavefunction, generalizing Bose and Fermi statistics, when two of them are interchanged. This can be used to perform quantum computations [1]. We show how to simulate the creation and manipulation of Abelian and non-Abelian anyons in topological lattice models using trapped atoms in optical lattices. Our proposal, feasible with present technology, requires an ancilla particle which can undergo single particle gates, be moved close to each constituent of the lattice and undergo a simple quantum gate, and be detected.
\end{abstract}

PACS numbers: 03.67.Lx, 03.65.Vf, 37.10.Jk

The quest for physical systems where anyons [2] can be observed has concentrated so far in effectively $2 d$ materials exhibiting topological order [3]. Abelian anyons, whose interchange generates a nontrivial phase in the wavefunction, exist in the Fractional Quantum Hall effect. Non-Abelian anyons, whose interchange effects full unitary gates on the wavefunction, are expected at certain filling fractions [4] (see recent experimental progress in [5]). In spin lattice systems, anyons can appear as low-lying excitations of topologically ordered ground states (see, e.g., $[1,6,7])$. Several implementations of lattice models with anyonic excitations have been put forward [8][9][10][11][12][13][14]. Those involving atoms or molecules in optical lattices are especially attractive, given recent experimental progress [15]. Specifically, Kitaev's honeycomb lattice model [16] can be engineered [11, 12], and anyonic interferometry in its Abelian phase can be performed with cavity-mediated global string operations [13] or using individual addressing to braid excitations [17] (but due to the perturbative nature of the effective Hamiltonian in this model, the visibility of anyonic interferometry is degraded $[13,18]$.)

Here we propose a novel scheme to create topologically ordered states, generate and braid anyons, and detect their statistics for any setup based on particles in optical lattices. We use a lattice of particles of species A to build the topological code and an ancilla of different species B that can be moved independently and brought close to any A particle to perform controlled operations on the code [19]. Preparing the ancilla in superposition states, making it interact with appropriate code particles and measuring its state, the following can be achieved: creation of a topological state, or a general errorcorrecting code (ECC); creation, braiding, and measurement (fusion) of anyons, all operations needed to perform topological quantum computation (TQC) by braiding; and anyonic interferometry, allowing direct observation of anyonic statistics. Note that (i) by using an ancilla with different quantum states to perform the manipulation of the anyons, tasks can be carried that are not possible using classical (e.g., laser) manipulation of anyons (without single-particle addressability, all proposed methods lack the power of ours); (ii) there is no need in principle of single-particle addressability, specially to perform proof-of-principle experiments; (iii) it is based on successfully demonstrated technologies [20][21][22]; (iv) it is the first realistic protocol for simulating universal TQC in an atomic, molecular, optical system system [while engineering the microscopic Hamiltonian to build topological protection may be some time off (though see [23]), the method herein works independently of the existence of the background Hamiltonian.]

We consider $2 d$ lattices loaded with atoms or molecules, e.g., ${ }^{87} \mathrm{Rb}$. The ancilla, e.g., ${ }^{23} \mathrm{Na}$, can be moved independently using a laser potential not affecting $\mathrm{Rb}$ atoms (see Fig. 1.) These are now routinely loaded in optical lattices, and in the Mott insulator state one can have extended regions with one particle per site [22, 24]. Single particles can also be loaded in optical potentials and moved without decoherence [21]. Our scheme can be extended to layered $3 d$ configurations [25]. We first consider the toric code Hamiltonian [1], with Abelian anyonic excitations only, as a toy model, but our scheme is basically model independent; later we apply it to the $\mathrm{D}\left(S_{3}\right)$ quantum double model [1], which has non-Abelian anyons and is universal for TQC [26].)

When the ancilla is brought close to a code atom, they experience a 2-qubit unitary $U_{Z}=|0\rangle_{a}\langle 0|\otimes I+| 1\rangle_{a}\langle 1| \otimes Z$ between their internal levels $(X, Y, Z$ are Pauli operators.) This gate can be implemented by cold collisions [27] or any other means $[28,29]$. Single qubit operations can be applied to the ancilla without having to address it, due to the different level structure of code atoms. Gates like $U_{X}=|0\rangle_{a}\langle 0|\otimes I+| 1\rangle_{a}\langle 1| \otimes X$ can be implemented by applying appropriate gates before and after $U_{Z}$. The internal ancilla state (measurement of $Z_{a}$ ) can also be detected with standard techniques without having to address it or affect the code.

We next show how to create Kitaev's toric code [1] with these tools. The code is defined as the ground level of a stabilizer Hamiltonian on a square lattice of qubits, realized as $\mathrm{Rb}$ atoms, at the edges of a square lattice. The Hamiltonian $H=-\sum_{\mathrm{v}} A_{\mathrm{v}}-\sum_{\mathrm{p}} B_{\mathrm{p}}$ is the sum of mutually commuting stabilizers $A_{\mathrm{v}}=\prod_{i \in \mathrm{v}} X_{i}$ and $B_{\mathrm{p}}=\prod_{i \in \mathrm{p}} Z_{i}$, where v runs over all vertices and p over plaquettes and products involve 


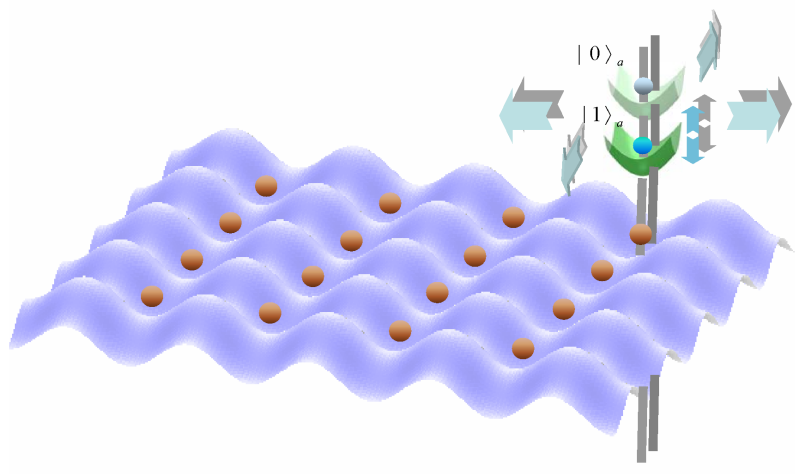

FIG. 1: $2 d$ optical lattice in the $x-y$ plane loaded with one atom per site. Atoms are in different Zeeman levels $|0\rangle$ and $|1\rangle$ in their ground electronic state, storing quantum information. An ancillary atom of a different species in its electronic ground state, with relevant Zeeman levels $|0\rangle_{a}$ and $|1\rangle_{a}$, is trapped using laser standing waves along the three directions. Lasers in the $x-y$ plane are far detuned from the fine structure splitting: The potential controlling horizontal movement of the ancilla does not depend on its internal state. The laser propagating along the $z$ direction is tuned in between the fine-splitted excited $P$ levels: The potential controlling the vertical movement depends on the internal state $[27,28]$.

the qubits surrounding the vertices or plaquettes. We can associate the presence or absence of particle-like excitations at the plaquettes (magnetic defects) and vertices (electric defects) with the fulfillment or not of the ground level conditions $A_{\mathrm{v}}=+1$ and $B_{\mathrm{p}}=+1$. Plaquette and vertex excitations are thus characterised by $B_{\mathrm{p}}=-1$ and $A_{\mathrm{v}}=-1$ and appear at the ends of strings of $X$ and $Z$ operators applied on a ground state. These 'particles' turn out to have nontrivial (anyonic) exchange statistics due to the anticommutation of the $X$ and $Z$ Pauli operators, namely the wavefunction gets multiplied by -1 when a vertex particle winds around a region containing a single plaquette excitation. Detection of this phase change is possible via interference experiments involving superpositions of states with and without anyons. Moreover, the degeneracy of the code allows to interpret it in terms of a set of logical qubits whose $Z$ and $X$ operators are given in terms of chains of $Z$ 's and $X$ 's.

We work with a rectangular surface with smooth and rough boundaries [25], with appropriate 3-body vertex and plaquette operators along the boundary providing for a two-dimensional code space: One logical qubit is encoded as the eigenvalue of a chain of Z's along an edge path connecting the rough boundaries. The code space is spanned by +1 coeigenstates of the stabilizers. To create a state $|\Psi\rangle$ in the code, we start with a well defined state $|0\rangle^{\otimes N}\left(+1\right.$ eigenstate of each $\left.B_{\mathrm{p}}\right)$ and measure the $A$ stabilizers sequentially, from left to right and top to bottom, using the ancilla. If all outcomes are +1 , our goal is achieved, since $|\Psi\rangle \propto \prod_{\mathrm{v}}\left(1+A_{\mathrm{v}}\right)|0\rangle^{\otimes N}[30]$. If -1 is obtained, we can correct by applying $Z_{b}$ to qubit $b$ at the bottom of the vertex using again the ancilla, since
$Z_{b}\left(1-A_{\mathrm{v}}\right)|0\rangle^{\otimes N}=\left(1+A_{\mathrm{v}}\right)|0\rangle^{\otimes N} ; Z_{b}$ is applied to a qubit that has not been measured yet (for the last row, $Z_{b}$ can be applied to the rightmost qubit.) Once we have measured all vertices and thus prepared the state, we can measure all stabilizers to detect errors and apply error-correcting $X$ 's or $Z$ 's to the corresponding qubits by using the ancilla. We could have started at another state, measured all operators in any order, and then corrected errors in this way to prepare the desired state (this can be used to prepare the target state in models beyond Kitaev's.) We now show how plaquette and vertex measurements, as well as $X$ 's and $Z$ 's, are performed using the ancilla. To measure $A_{\mathrm{v}}$, we prepare the ancilla in state $|+\rangle_{a} \propto|0\rangle_{a}+|1\rangle_{a}$, move it to each qubit in the vertex, and apply $U_{X}$ each time. Then we apply a Hadamard gate to the ancilla and measure $X_{a}$. If the result is \pm 1 , we have applied $\left\langle \pm\left|\prod_{i \in \mathrm{v}} U_{X}\right|+\right\rangle=\left(1 \pm A_{\mathrm{v}}\right)$ to the qubits at the vertex, thus performing the desired measurement. $B_{\mathrm{p}}$ is measured by substituting $U_{Z}$ for $U_{X}$. To apply $X(Z)$ to a qubit, we prepare the ancilla in state $|1\rangle_{a}$, approach it to that qubit and apply $U_{X}$ $\left(U_{Z}\right.$.) Once the toric code state is prepared, operations within the code are performed by applying strings of operators, using the ancilla in state $|1\rangle_{a}$, and applying $U_{X}$ or $U_{Z}$ sequentially on the desired qubits by bringing them close to the ancilla. To measure string operators, prepare the ancilla in state $|+\rangle$, follow the same sequence, and measure $X_{a}$ at the end. The toric code has two kinds of elementary excitations [1]: pairs of anyons in frustrated vertices (electric defects, $A_{\mathrm{v}}=-1$ ) and in frustrated plaquettes (magnetic defects, $B_{\mathrm{p}}=-1$ ), with mutual Abelian anyonic statistics. They can be created by applying $Z$ or $X$ to a given qubit, and can be moved, braided, and fused together by applying these operators along a given path using the ancilla. Superpositions of states with and without vortices, or where they are in different places (see Fig. 2), can be created, allowing the observation of fractional statistics: The simplest interference experiment is shown in Fig. 3. On how to infer anyonic statistics from interference experiments, see the Appendix.

We now outline the preparation and manipulation of anyons in a non-Abelian setting universal for quantum computation [26]: the lattice quantum double model $\mathrm{D}\left(S_{3}\right)$ based on the group of permutations of 3 elements $S_{3}$ [1] (see a brief discussion in the Appendix; the full construction is given in [31].) It is a lattice model generalizing the toric code, where local degrees of freedom live at the (oriented) edges of the lattice with orthonormal bases $\{|g\rangle\}$ labeled by the six group elements $g \in$ $G$. The Hamiltonian, also of the form $H=-\sum_{\mathrm{v}} A_{\mathrm{v}}-\sum_{\mathrm{p}} B_{\mathrm{p}}$, has commuting vertex and plaquette stabilizers imposing constraints on the ground states. Their violations define particlelike excitations (anyons) with topological charges (electric, magnetic and dyonic), with non-Abelian fusion and braiding rules. Creation, transport, and fusion of anyons can be achieved generalizing the controlled-NOT operations of the toric code to controlled left and right group multiplications: $\mathcal{U}_{h}^{\mathrm{L}, \mathrm{R}}=|0\rangle_{B}\left\langle 0\left|\otimes I_{A}+\right| 1\right\rangle_{B}\langle 1| \otimes\left(\sigma_{h}^{\mathrm{L}, \mathrm{R}}\right)_{A}$ with $\sigma_{h}^{\mathrm{L}}|g\rangle=|h g\rangle$, $\sigma_{h}^{\mathrm{R}}|g\rangle=|g h\rangle$. The local degrees of freedom for the $\mathrm{D}\left(\mathrm{S}_{3}\right)$ model are qudits of six dimensions, and their six basis ele- 

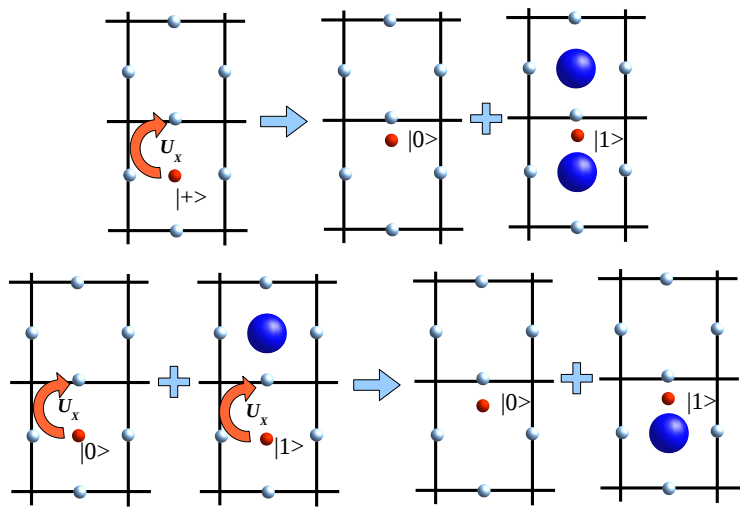

FIG. 2: Top: Creation of a superposition of the vacuum and a pair of magnetic defects. On top of the ground state (code atoms shown as light circles), an ancilla (dark) is initialised as $|+\rangle$ and brought close to a code atom, effecting $U_{X}=|0\rangle\langle 0|\otimes I+| 1\rangle\langle 1| \otimes X$ and creating two magnetic defects (big blobs) in the adjacent plaquettes in the superposition component where the ancilla is in state $|1\rangle$, while the code remains in the ground state in sector $|0\rangle$. Bottom: Anyon transport. The ancilla interacts via $U_{X}$ with a code atom between a plaquette satisfying the ground state condition and one of the plaquettes containing a magnetic defect in a superposition sector, transferring the anyon to the first plaquette.

ments can be encoded into ground electronic hyperfine states of an alkali atom with enough levels, used as code lattice A. To create, transport, and fuse pure electric and magnetic charge states (enough to simulate universal TQC [26]), a 6-state ancilla species B is especially appropriate (see Fig. 4.) As in the toric code, vertex operators can be measured using ancillaassisted operations to prepare the ground state (see the Appendix.) With one single ancilla, which need not be spatially addressed, our algorithm requires $O(\mathrm{~nm})$ steps in an $n \times m$ region; with an auxiliary lattice with one ancilla per face of the code lattice, assuming addressability, it can be parallelized to depth $O(n+m)$ (essentially optimal [32]; see the Appendix.)

This scheme is independent of the method used to construct the topological state. It can be built by cooling an atomic ensemble interacting via an engineered topological Hamiltonian, providing in principle topological protection to the code except for anyonic manipulations, which should take the system to excited levels in a controlled way (as needed to perform TQC as such; on how to simulate relevant Hamiltonians, see $[11,12]$.) But it can also be constructed by the above procedure using ancillas to impose stabilizer constraints, enough to perform proof-of-principle interference experiments, a worthy goal by itself. This also allows fault-tolerant quantum computation with general ECCs (topological codes are excellent ECCs: local operators do not mix topological sectors, string operators mixing them can be efficiently implemented.)

The arrangement of ancillas is flexible. One ancilla, individually manipulable [21], suffices in principle. Varying degrees of parallelization are possible: the ground state can
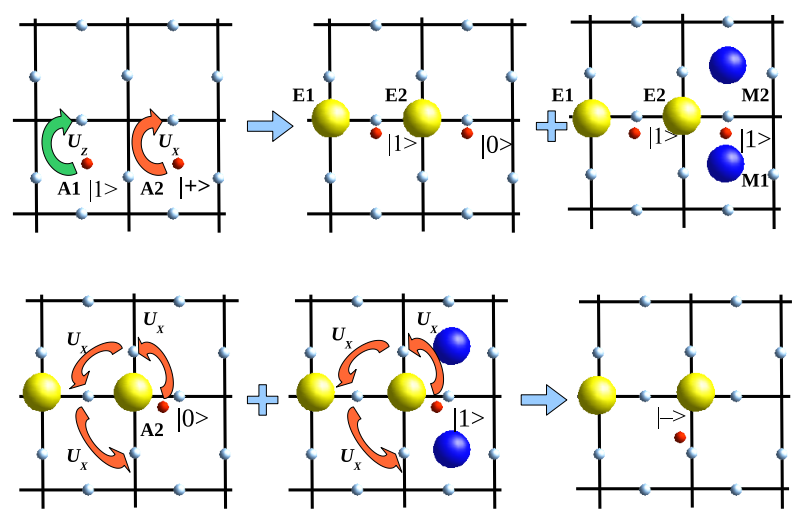

FIG. 3: Minimal interferometry experiment for the toric code. Top: Ancilla A1, initialised as $|1\rangle$, unconditionally creates a pair E1, E2 of electric defects at neighboring vertices by application of $U_{Z}=$ $|0\rangle\langle 0|\otimes I+| 1\rangle\langle 1| \otimes Z$. Ancilla A2, initialised as $|+\rangle$, creates a superposition of the presence and the absence of two magnetic defects M1, M2 at neighboring plaquettes by application of $U_{X}$. Bottom: M1 is wound around E2 by sequential $U_{X}$ interaction of ancilla A2 with the code atoms surrounding E2. M1 and M2 are eventually reannihilated, bringing both sectors to the ground state with a relative minus sign, $|\mathrm{GS}\rangle_{\text {code }} \otimes|1\rangle_{\mathrm{A} 1} \otimes|-\rangle_{\mathrm{A} 2}$, i.e., a phase -1 is generated in the sector where braiding of defects takes place. In this case, the interferometry results can be read from the ancilla lattice by a local projective measurement on A2.

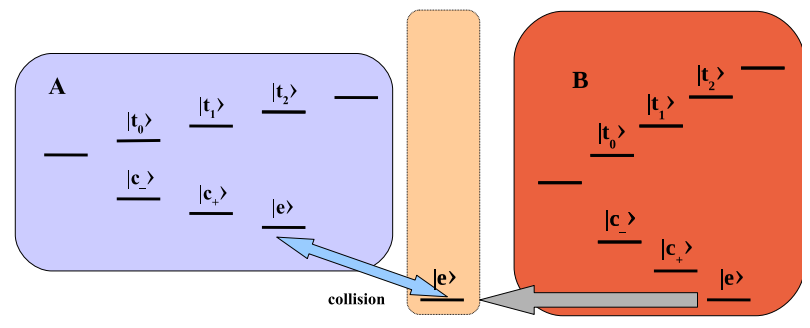

FIG. 4: Level structure for the $\mathrm{D}\left(\mathrm{S}_{3}\right)$ model. The elements of $S_{3}$ are encoded into ground electronic hyperfine states of a trapped alkali atom $\mathrm{A}\left({ }^{87} \mathrm{Rb}\right.$ or ${ }^{23} \mathrm{Na}$, with 8 levels, or ${ }^{133} \mathrm{Cs}$, with 16 levels.) A 6-state ancilla $B$ of a different species is used to control operations. Ancilla states can be moved independently: bringing $|e\rangle_{B}$ close to a code atom $\mathrm{A}$ and coupling it to $|e\rangle_{A}$, a collisional phase gate $Z_{e}=I_{A} \otimes I_{B}-2|e\rangle_{B}\langle e|\otimes| e\rangle_{A}\langle e|$ is obtained; together with simultaneous 1-particle operations $\mathcal{V}_{\text {all A }}=\bigotimes_{A} v_{A}$ on the code, it provides all controlled operations. Indeed, $\mathcal{V}_{\text {all a }}^{\dagger} z_{e} \mathcal{V}_{\text {all a }}$ yields controlled phase gates for any code state with $v_{A}=|g\rangle_{A}\langle e|+| e\rangle_{A}\langle g|$, and transpositions with $v_{A} \propto(|e\rangle+|g\rangle)_{A}\left\langle e\left|+(|e\rangle-|g\rangle)_{A}\langle g|\right.\right.$ (these can be composed to obtain any controlled group multiplication.)

be constructed with sequential operations on one column of the sample (parallel to smooth boundaries) at a time, so as to cover the whole sample; in interferometry, commuting operations can be done simultaneously; some parallelization can be introduced with a coarser optical potential for the ancilla than for the code.

This method shares common problems of optical lattice schemes of quantum computation, in particular, spontaneous emission. Essentially, only the vertical direction of the ancilla 
is close to resonance; lifetimes of seconds can be reached by tuning the laser between the fine structure levels and can be enhanced by restricting manipulation of the vertical dynamics to the (short) times the ancilla is close to a code atom. The ancilla can be repumped after an operation, allowing one to repeat the tasks and detect errors. Controlled logic by cold collisions [27] requires cooling the system to the physical ground state, possible for both the code (in the 1st Bloch band, the ground state of the local potential) and the ancilla. Rydberg gates [28, 29] based on dipole-dipole interactions eliminate this condition. Ancilla-code interactions must break the code in a controlled way, not creating (superpositions of) stray anyons spoiling the quantum memory: Theoretical analysis [33] and experimental results [34] suggest that excellent control and small decoherence rates are achievable. Then the implementation benefits from the added protection of topological codes (or, in general, ECCs.) With a bias magnetic field, arbitrary single qudit unitaries can be realized using frequency and polarization selectivity of microwave or Raman laser pulses. Collisional gates can be realized using trapinduced shape resonances [35], or using Raman pulses to map code and ancilla ground states $\left(|e\rangle_{A, B}\right.$ in figure 4) to a vibrational excited state of each lattice well, evolving by a collisional phase and mapping back [36].

The experimental techniques required by our method are (i) independent trapping of two particle species A and B with different laser trapping potentials; (ii) diluting the population of species B so that each particle is individually addressable; (iii) bringing species A to a Mott insulator phase; (iv) initialisation of species A in a product state $|0\rangle^{\otimes N}$; (v) single particle gates on species B; (vi) simultaneous gates on all particles of species A; (vii) independent transport of internal states of single particles of species B so as to effect cold collisions with particles of species A. Additionally, large scale simulations would require the ability to recool to vibrational states. Each one of these techniques has been demonstrated experimentally; bringing them together will pose an interesting experimental challenge.

A method based on the availability of an ancilla species thus allows one to perform all TQC tasks on a given topological state, independently of the way in which this state is constructed, in optical lattices; e.g., universal TQC based on braiding can be performed on top of the ground state of the $\mathrm{D}\left(\mathrm{S}_{3}\right)$ model. This scheme is likely to prove the most practical and general way to perform TQC in optical lattices. Large computations will face a steep scaling (a problem not exclusive of topological settings), but observing interference phenomena and applying gates by anyon braiding is feasible with today's technology. This method can also be used advantageously in general ECCs.

Acknowledgements: Financial support from the EU (SCALA) and DFG (MAP and NIM Excellence Clusters) is gratefully acknowledged. M. A. thanks S. Iblisdir, D. PérezGarcía and J. Pachos for discussions on the quantum double models.

\section{APPENDIX: SUPPLEMENTARY MATERIAL}

In this Appendix we describe in some detail how to simulate non-Abelian anyonic models. Our construction is based on a generalization of the toric code which is spin lattice realization of the quantum double $D(G)$ of a discrete group $G$. To realize this we work on a connected two complex $\Gamma=\{\mathcal{V}, \mathcal{E}, \mathcal{F}\}$ which is a cellulation of a two dimensional surface described by vertex, edge, and face sets. Particles with $d=|G|$ levels (qudits) are placed on the edges and physical states live in a Hilbert space $\mathcal{H}=\mathcal{H}(d)^{\otimes|\mathcal{E}|}$ where $\mathcal{H}(d)=\{\mathbb{C}|j\rangle\}_{j=0}^{d-1}$. As in the toric code, particles on edges that meet at a vertex $v$ all interact via a vertex operator $A(v)$. Similarly, all particles on edges that are on the boundary of a face $f$ interact via $B(f)$. We pick an orientation for each edge with $e=\left[v_{j}, v_{k}\right]$ denoting an edge with arrow pointing from vertex $v_{j}$ to $v_{k}$. The choice of edge orientations is not important as long as a consistent convention is used. The face orientations are all chosen consistent with the surface orientation. Whereas in the toric code model, the ground states are invariant under local $\mathbb{Z}_{2}$ gauge transformations generated by $\{A(v)\}$, here we demand gauge invariance under transformations:

$$
T_{g}(v)=\prod_{e_{j} \in[v, *]} L_{g}\left(e_{j}\right) \prod_{e_{j} \in[*, v]} R_{g^{-1}}\left(e_{j}\right),
$$

where $L_{g}\left(e_{j}\right), R_{g}\left(e_{j}\right) \in U(d)$, the $d$ dimensional unitary group, are the permutation representations of the left and right action of multiplication by the group element $g \in G$ on the system particle located at edge $e_{j}$. For the particle states we make the identification $|j\rangle \equiv\left|g_{j}\right\rangle$, where by convention $|0\rangle \equiv\left|g_{0}\right\rangle \equiv|e\rangle$, with $e$ the identity element. The action of left and right group multiplication on the basis states is then $L_{h}|j\rangle=\left|h g_{j}\right\rangle, R_{h}|j\rangle=\left|g_{j} h\right\rangle$. A suitable spin lattice model was introduced by Kitaev [1]:

$$
H_{\mathrm{TO}}=-\sum_{v} A(v)-\sum_{f} B(f)
$$

where

$$
\begin{aligned}
& A(v)=\frac{1}{|G|} \sum_{g \in G} T_{g}(v) \\
& B_{\ell}(v, f)=\sum_{\left\{\prod_{e_{k} \in \partial f} g_{k}^{-o_{f}\left(e_{k}\right)}=\ell \mid e_{0}=[v, * *\}\right\}} \otimes_{e_{k} \in \partial f}\left|g_{k}^{-o_{f}\left(e_{k}\right)}\right\rangle e_{k}\left\langle g_{k}^{-o_{f}\left(e_{k}\right)}\right|,
\end{aligned},
$$

The operator $A(v)$ is a projector onto gauge symmetrized states. Similarly, the operator $B_{\ell}(v, f)$, is a projector onto states with flux $\ell$ at face $f$. Here the sum is taken over all products of group elements $g_{k}$ acting on a counterclockwise cycle of edges on the boundary of $f$ such that the product is $\ell \in G$. The function $o_{f}\left(e_{j}\right)= \pm 1$ according to whether the orientation of the edge is the same as (opposite to) the face orientation. Assigning the origin $v$ from which the product is taken is important as failure to do so is equivalent to only specifying the conjugacy class or magnetic charge. Since the trivial flux 
is its own conjugacy class we write it $B_{e}(v, f)=B(f)$, which is the projection we seek for ground states of $H_{T O}$.

By construction $\left[A(v), A\left(v^{\prime}\right)\right]=\left[B(f), B\left(f^{\prime}\right)\right]=$ $[A(v), B(f)]=0$. The ground states of $H_{\mathrm{TO}}$ are then manifestly gauge invariant states defined as +1 coeigenstates of $\{A(v), B(f)\}$. Excited states are described by violations of the local constraints $A(v), B(f)$ and are particle-like corresponding to irreducible representations (irreps) $\Pi_{R\left(N_{[\alpha]}\right)}^{[\alpha]}$ where $[\alpha]$ labels the magnetic charge denoting a conjugacy class of $G$, and $R\left(N_{[\alpha]}\right)$ labels the electric charge which denotes a unitary irrep $R$ of the normalizer of an element in the conjugacy class $[\alpha]$. Notice that for the group $\mathbb{Z}_{2}$, $L_{e}=R_{e}=\mathbf{1}_{2}$ and $L_{g_{1}}=R_{g_{1}}=\sigma^{x}$, and $H_{T O}$ is precisely the toric code Hamiltonian.

Henceforth, we fix $G=S_{3}=\left\{g_{j}\right\}_{j=0}^{5}$ the symmetric group of three objects and let $\Gamma$ be an $n \times m$ square lattice with boundary. We place $d=6$ qudits on edges labeling each by it's location $e_{i, j ; k, l}=\left[v_{i, j}, v_{k, l}\right]$ and $d=6$ vertex and face ancillas labeled by their locations $v_{j, k}$ and $f_{j, k}$. For a two complex with boundary the ground state $|G S\rangle$ of $H_{T O}$ is unique [37] and can be constructed by measuring the vertex projectors in an analogous way to the toric code. We begin with all system particles in state $|e\rangle=|0\rangle$ which satisfies the zero flux condition $\langle B(f)\rangle=1 \forall f$. All vertex and face ancillae are prepared in the state $|\tilde{0}\rangle$ where $|\tilde{j}\rangle=\frac{1}{\sqrt{6}} \sum_{k=0}^{5} e^{2 \pi i j k / 6}|k\rangle$. We then apply a sequence of operations columnwise beginning on the left column of vertices $\left\{v_{k, 0}\right\}$ and ending on the right. For column $k$ we apply the controlled operation $W(v)=\sum_{h \in S_{3}}|h\rangle_{v}\langle h| \otimes T_{g}(v)$ between each vertex ancilla $v_{j, k}$ and its edge neighbors. These operations can be done in parallel since $\left[L_{h}(e), R_{h^{\prime}}(e)\right]=0$. Next we measure each vertex ancilla $v_{j, k}$ in the basis $\{|\tilde{r}\rangle\}$ and given the outcome $m(j, k)$ apply the single qudit correction gate $Z^{m(j, k)}\left(e_{j, k ; j, k+1}\right)$, where $Z(e)=\sum_{k=0}^{5} e^{2 i \pi k / 6}|k\rangle_{e}\langle k|$, on the right edge. Finally when we reach the rightmost column of vertices $\left\{v_{j, m-1}\right\}$ we apply a sequence of operations from bottom to top involving applying $W\left(v_{j, m-1}\right)$ followed by measurement of the ancilla and the correction gate $Z^{m(j, m-1)}\left(e_{j, k ; j, k+1}\right)$ on the top edge. When we reach vertex $v_{n-1, m-1}$ we are done since the operator $T\left(v_{n-1, m-1}\right)$ is not independent but can be written as a product of the others meaning that $\left\langle A\left(v_{n-1, m-1}\right)\right\rangle=1$ already.

Our algorithm has a computational depth of $O(m+n)$ as measured by the number of parallel elementary two qudit gates. We find contructions of the operators $\sum_{h \in S_{3}}|h\rangle\langle h| \otimes L_{h}$ or $\sum_{h \in S_{3}}|h\rangle\langle h| \otimes R_{h}$ in 37 controlled phase gates $e^{i \pi|0\rangle\langle 0|\otimes| 0\rangle\langle 0|}$ and roughly twice that number of local pairwise basis state couplings and suspect this count is optimal. One might wonder if a faster ground state preparation procedure is possible. The answer is no if the initial state is uncorrelated and the available set of operations is quasi-local. The reason is that the final state has global correlations that are created by quasi-local operations. In our algorithm these operations are measurements but they could also be adiabatic turn on of the summands of $H_{T O}$. The time scale to perform the quasi-local operations (here the measurement time of $A(v)$ ) establishes a light cone for the flow of correlations. In [32] it was shown by an application of the Lieb-Robinson bound that the minimal time to prepare a topologically ordered state beginning in a completely uncorrelated state is of the order of the length of the correlations. Since the correlation length scales as the linear dimension of our lattice, our algorithm is essentially optimal.

The group $S_{3}$ has three conjugacy classes: the identity $[e]=\{e\}$, transpositions $[t]=\left\{t_{0}, t_{1}, t_{2}\right\}$ and cyclic permutations $[c]=\left\{c_{+}, c_{-}\right\}$, and three irreps: the two one dimensional irreps $R_{1}^{+}(g)=1$, the signature representation

$$
R_{1}^{-}(e)=+1=R_{1}^{-}\left(c_{\rho}\right), \quad R_{1}^{-}\left(t_{i}\right)=-1,
$$

and the two-dimensional irrep

$$
R_{2}(e)=\mathbf{1}_{2}, \quad R_{2}\left(t_{k}\right)=\sigma^{x} e^{i \frac{2 \pi}{3} k \sigma^{z}}, R_{2}\left(c_{\rho}\right)=e^{i \rho \frac{2 \pi}{3} \sigma^{z}} .
$$

For $D\left(S_{3}\right)$ there are 8 irreps: the vacuum state $\Pi_{R_{1}^{+}}^{[e]}$, pure magnetic charges $\Pi_{\beta_{0}}^{[c]}, \Pi_{\gamma_{0}}^{[t]}$, pure electric charges $\Pi_{R_{1}^{-}}^{[e]}, \Pi_{R_{2}}^{[e]}$, and dyonic combinations $\Pi_{\beta_{1}}^{[c]}, \Pi_{\beta_{2}}^{[c]}, \Pi_{\gamma_{1}}^{[t]}$. A complete derivation of the fusion rules and braid relations for this model is given in [38]. We focus here pure electric or pure magnetic charge states, which in fact are sufficient for universal topological quantum computation [26].

Pure electric charges are labeled by basis states $\left|\left(P_{\mu, v}^{R}, e\right) ;(v,-)\right\rangle$ where $P_{\mu, v}^{R}=\frac{|R|}{|G|} \sum_{g \in G}\left[R(g)^{*}\right]_{\mu, v} g$ is the projection operator onto a subspace belonging to the unirep $R$. Since we are considering pure electric charges, the unireps of the normalizer of $[e]$ are equivalent to the unireps of $G$ itself. In the context of the spin lattice model this is interpreted as electric charge created by applying the projection operator $P_{\mu, \nu}^{R}$ onto the system with the group action being local gauge transformations $T_{g}(v)$. Magnetic fluxes are labeled by basis states: $\left|\left(P^{R_{1}^{+}}, \ell\right) ;(v, f)\right\rangle$ and are understood as the result of a projection $B_{\ell}(v, f)$.

Excitations created in the bulk always come in particle anti-particle pairs. A generic state of a magnetic charge pair $\left(\left[\ell, \ell^{-1}\right]\right)$ is

$$
\sum_{\ell \in[\ell]} c_{\ell}\left|\left(P^{R_{1}^{+}}, \ell^{-1}\right) ;\left(v_{i, j}, f_{i, j}\right)\right\rangle\left|\left(P^{R_{1}^{+}}, \ell\right) ;\left(v_{i, j}, f_{i, j+1}\right)\right\rangle
$$

with $\sum_{\ell \in[\ell]}\left|c_{\ell}\right|^{2}=1$. The unique vacuum magnetic charge pair state invariant under conjugation by fluxes is the state with $c_{\ell}=1 / \sqrt{|[\ell]|} \forall \ell$, denoted $\left|0_{[\ell]} ;(v, f),\left(v^{\prime}, f^{\prime}\right)\right\rangle$. Note the vacuum state with neighboring magnetic charge pairs can be written

$$
\left|0_{[\ell]} ;\left(v_{i, j}, f_{i, j}\right),\left(v_{i, j}, f_{i, j+1}\right)\right\rangle=\frac{1}{\sqrt{|[\ell]|}} \sum_{\ell \in[\ell]} R_{\ell}\left(e_{i-1, j ; i, j}\right)|G S\rangle
$$

We can prepare this by beginning with the ancilla $f_{i, j}$ in the state $\left|0_{[\ell]}\right\rangle_{f_{i, j}}$ where $\left\{\left|k_{[\ell]}\right\rangle=Z_{[\ell \ell]}^{k}\left|0_{[\ell]}\right\rangle\right\}_{k=0}^{|[\ell]|-1}$, with $Z_{[\ell]}^{k}=$ 
$\sum_{\ell_{m} \in[\ell]} e^{i 2 \pi k m /|[\ell]|}\left|\ell_{m}\right\rangle\left\langle\ell_{m}\right|$ and we have labeled the group elements in $\left.[\ell]=\left\{l_{0}, \ldots, \ell_{|[\ell]|-1}\right\}\right)$, and applying the two qudit unitary

$$
F_{[\ell]}\left(f_{i, j}\right)=\mathbf{1}_{|G|-|[\ell]|} \otimes \mathbf{1}_{|G|}+\sum_{\ell \in[\ell]}|\ell\rangle_{f_{i, j}}\langle\ell| \otimes R_{\ell}\left(e_{i-1, j ; i, j}\right)
$$

Next we measure the face ancilla in the basis $\left\{\left|k_{[\ell]}\right\rangle\right.$. For the outcome $0_{[\ell]}$ the target magnetic charge state is created. Otherwise for outcome $k_{[\ell]}$, we need a correction step. To do this prepare the ancilla $f_{i, j}$ in the state $|e\rangle_{f_{i, j}}$ and apply the controlled operation $\Lambda\left(v_{i, j}, f_{i, j}\right)$ where

$$
\Lambda(v, f)=\sum_{g \in G} B_{g}(v, f) \otimes L_{g}(f) .
$$

which maps the ancilla $f$ to state $|g\rangle_{f}$ when the flux at $f$ evaluated with base point $v$ is $g$. Such a controlled operation can be decomposed into elementary two qudit controlled rotation operators with each edge $e_{k}$ surrounding $f$ as a control and the ancilla as the target.

In the toric code and in fact for discrete gauge theories for all finite Abelian groups [37], excitations can be propagated by applying one local operator which simultaneously annihilates a charge at one location and create one at a neighboring location. This is not true for non-Abelian theories as doing so violates a face or vertex constraint. To propagate magnetic charges from one face $f$ to an adjacent face $f^{\prime}$ essentially involves coherently mapping the value of flux at $f$ to the face ancilla $f$ using $\Lambda(v, f)$ and applying a controlled operation on the shared edge of the faces $f, f^{\prime}$. After this controlled operation the face ancilla $f$ is disentangled from the system by mapping the flux at face $f^{\prime}$ to ancilla $f^{\prime}$ and performing a controlled operation between ancillae $f, f^{\prime}$ and finally reversing the mapping on $f^{\prime}$. In this protocol we are careful to demand only single qudit and nearest neighbor two qudit interactions. This entire process respects superpositions over flux states and can therefore be used to propagate magnetic charges around the lattice [31]. Fusion of a magnetic charge pair can be measured by using controlled operations to bring the constituent charges in conjugacy class $[\ell]$ adjacent to one another at faces $\left(f, f^{\prime}\right)$ and applying the operator $\Lambda(v, f)=\sum_{g \in G} B_{g}(v, f) \otimes L_{g}(f)$ followed by measurement of the ancilla $f$ in the basis $\left|0_{[\ell]}\right\rangle f_{i, j}$. The probability to obtain the outcome $0_{[\ell]}$ equals the probability for the pair to fuse into the vacuum.

A generic state of an electric charge pair $\left(R, R^{*}\right)$ at vertices $\left(v, v^{\prime}\right)$ will be represented as an $|R| \times|R|$ matrix

$$
\begin{aligned}
\left|M^{R} ;\left(v, v^{\prime}\right)\right\rangle & =\frac{1}{\sqrt{|R|}} \sum_{\mu, v} M_{\mu, v}^{R} \\
& \times \frac{1}{\sqrt{|R|}} \sum_{\beta=0}^{|R|-1}\left|\left(P_{\mu, \beta}^{R}, e\right) ;(v,-)\right\rangle\left|\left(P_{v, \beta}^{R *}, e\right) ;\left(v^{\prime},-\right)\right\rangle
\end{aligned}
$$

with $\sum_{\mu, v=0}^{|R|-1}\left|M_{\mu, v}^{R}\right|^{2}=|R|$. There is a unique vacuum electric charge state which is invariant under conjugation by fluxes:
$\left|\mathbf{1}^{R} ;\left(v, v^{\prime}\right)\right\rangle$. The state $\left|\mathbf{1}^{R} ;\left(v_{i, j}, v_{i, j+1}\right)\right\rangle$ can be prepared as follows. First prepare the vertex ancilla $v_{i, j}$ in state $|e\rangle_{v_{i, j}}$. and apply the conditional unitary $K\left(v_{i, j}, e_{i, j ; i, j+1}\right)$ defined by

$$
K(v, e)=\left\{\begin{array}{cc}
\sum_{g \in G}|g\rangle_{e}\langle g| \otimes R_{g}(v) & e=[v, *] \\
\sum_{g \in G}|g\rangle_{e}\langle g| \otimes R_{g^{-1}}(v) & e=[*, v]
\end{array}\right.
$$

Depending on the representation, $R$, apply a single qubit operation $W_{R}$ on the ancilla $\left(v_{i, j}\right)$ :

$$
\begin{aligned}
& W_{R_{1}^{+}}=\mathbf{1}_{6} \\
& W_{R_{1}^{-}}=|e\rangle\left\langle e|+| c_{+}\right\rangle\left\langle c_{+}|+| c_{-}\right\rangle\left\langle c_{-}|-| t_{0}\right\rangle\left\langle t_{0}|-| t_{1}\right\rangle\left\langle t_{1}|-| t_{2}\right\rangle\left\langle t_{2}\right| \\
& W_{R_{2}}=2|e\rangle\left\langle e|-| c_{+}\right\rangle\left\langle c_{+}|-| c_{-}\right\rangle\left\langle c_{-}\right|
\end{aligned}
$$

(the latter is not unitary but can be constructed using adaptive measurements as detailed in [31]) and finally apply $K^{-1}\left(v_{i, j}, e_{i, j ; i, j+1}\right)$ to disentangle the ancilla from the system. One can prepare more distant vacuum charge pairs, e.g. $\left|\mathbf{1}^{R} ;\left(v_{i, j}, v_{i^{\prime}, j^{\prime}}\right)\right\rangle$ using the same procedure but after each application of $K(v, e)$, swapping the vertex qudit with the next relevant vertex qudit on the path (via an intermediary swap with the in between edge qudit) then applying the operator $U_{R}\left(v_{i^{\prime}, j^{\prime}}\right)$ at the end, and finally inverting the steps targeting the ancilla. Fusion of electric charge pair $\left|M^{R} ;\left(v, v^{\prime}\right)\right\rangle$ is measured by beginning at vertex $v$ and applying a sequence of controlled operations $K(v, e)$ acting on a path of vertices from $v$ to $v^{\prime}$. At vertex $v^{\prime}$, the operator $L_{c_{+}}\left(v^{\prime}\right)$ is applied and then the set of controlled operations along the vertex path is inverted. Finally, the first ancilla $v$ is measured in any unitary extension of the basis $\left\{|R\rangle=U_{R} \sum_{g \in S_{3}}|g\rangle / \sqrt{6}\right\}$. The probability to measure the outcome state $\left|R_{1}^{ \pm}\right\rangle$is the probability for fusion in the vacuum or into the signed irrep.

We now have all the steps to create and braid and fuse anyons. One can verify that all the usual rules for exchanging and braiding anyons are satisfied [31]. In particular it is easy to see that braiding charges around each other acts trivially since the electric charge pair creation operators are diagonal in the logical basis. Furthermore, the gauge transformations $T_{h}(v)$ can be viewed as creating a magnetic flux $h, h^{-1}$ pair, braiding them around the vertex $v$ and annihilating. To verify non trivial braiding, one can look for imperfect fusion of electric or magnetic charge pairs into the vacuum. Let's describe a simplified interference experiment that could be performed in principle on a single face of the lattice. We begin by preparing the state of adjacent electric charge pairs $\left|\mathbf{1}^{R_{2}} ;\left(v_{i, j}, v_{i, j+1}\right)\right\rangle$ and the vertex ancilla $v_{i, j}$ in $\left|h^{+}\right\rangle$where $\left|h^{ \pm}\right\rangle=(|e\rangle \pm|h\rangle) / \sqrt{2}$ for some $h \in S_{3}$. Next we apply the controlled operation $W\left(v_{i, j}\right)$ which creates the state $\left(|e\rangle_{v_{i, j}}\left|\mathbf{1}^{R_{2}} ;\left(v_{i, j}, v_{i, j+1}\right)\right\rangle+|h\rangle_{v_{i, j}}\left|\mathbf{1}^{R_{2}} ;\left(v_{i, j}, v_{i, j+1}\right)\right\rangle\right) / \sqrt{2}$ followed by measurement of the ancilla in the basis $\left|h^{ \pm}\right\rangle$with outcome $m= \pm 1$. The outcome distribution satisfies

$$
\begin{aligned}
P(m=1)-P(m=-1) & =\left|\left\langle\mathbf{1}^{R_{2}} ;\left(v, v^{\prime}\right) \mid R_{2}(h) ;\left(v, v^{\prime}\right)\right\rangle\right|^{2} \\
& =\frac{\left|\operatorname{Tr}\left[R_{2}(h)\right]\right|^{2}}{\left|R_{2}\right|^{2}}
\end{aligned}
$$

which is precisely the fusion probability for $R_{2}(h) \rightarrow \mathbf{1}^{R_{2}}$. 
It is important to clearly define what we mean by inferring anyonic statistics from interference measurements. For any physical theory, the phase accumulated when braiding one particle around another has contributions from the statistics $\phi_{s}$ as well as possible dynamical $\phi_{d}$ and geometric (Berry's phase) contributions $\phi_{g}$ [39]. Considering the interferometry experiment depicted in Fig. 3, including the other phases in the process gives the transformations $|G S\rangle|1\rangle_{A 1}|+\rangle_{A 2} \rightarrow\left|E 1, E 2 ;\left(v_{1}, v_{2}\right)\right\rangle|1\rangle_{A 1}|0\rangle_{A_{2}}+$ $|E 1, E 2\rangle|M 1, M 2\rangle|1\rangle_{A_{1}}|1\rangle_{A_{2}} \quad \rightarrow \quad|E 1, E 2\rangle|1\rangle_{A 1}|0\rangle_{A_{2}}+$ $e^{i\left(\phi_{s}+\phi_{g}+\phi_{d}\right)}|E 1, E 2\rangle|M 1, M 2\rangle|1\rangle_{A_{1}}|1\rangle_{A_{2}} \rightarrow|G S\rangle|1\rangle_{A 1}\left(|0\rangle_{A 2}+\right.$ $\left.e^{1\left(\phi_{s}+\phi_{g}+\phi_{d}\right)}|1\rangle_{A 2}\right) / \sqrt{2}$. If, e.g., there were a background Hamiltonian $H=-U \sum_{v} A_{v}-U \sum_{p} B_{p}$ present, then $\phi_{d}=t 4 U$ where $t$ is the time to perform the braiding. The statistical phase can be extracted by comparing this measured phase to a proper reference phase. To do so we can perform a similar set of operations but starting in the state $|G S\rangle|1\rangle_{A 0}|+\rangle_{A 2}$ such that the created electric defects neighboring A0 are not enclosed by the braiding path of the magnetic defects. Since the braiding paths and particle numbers in each component are the same in both experiments, the difference in the measured phases accumulated on $\mathrm{A} 2$ is $\phi_{s}$.

Our scheme is flexible in how the ancillary particles are introduced and one could work entirely within a single two dimensional lattice with in place face and vertex ancilla. For example, the retroreflected square lattice of [20] provides a bipartite square lattice with addressable systems and ancillary particles. By dynamically changing the trapping fields strengths and phases, the face and vertex ancilla can be moved along any cardinal direction to interact pairwise with the system particles on the edges. However, to build the ground states and manipulate anyons, individual addressability of the ancillary particles using, e.g., the techniques in [40, 41] seems necessary.

[1] A. Yu. Kitaev, Annals Phys. 303, 2 (2003), arXiv: quant-ph/ $9707021 \mathrm{v} 1$.

[2] F. Wilczek, Phys. Rev. Lett. 48, 1144 (1982); Phys. Rev. Lett. 49, 957 (1982).

[3] X.-G. Wen, Adv. Phys. 44, 405 (1995), arXiv:cond-mat/ 9506066v2.

[4] G. Moore and N. Read, Nucl. Phys. B360, 362 (1991).

[5] M. Dolev et al., Nature 452, 829 (2008), arXiv : 0802.0930v1 [cond-mat.mes-hall].

[6] M. A. Levin and X.-G. Wen, Phys. Rev. B71, 045110 (2005), arXiv: cond-mat/0404617v2 [cond-mat.str-el].

[7] P. Fendley and E. Fradkin, Phys. Rev. B72, 024412 (2005), arXiv: cond-mat/0502071v2 [cond-mat.str-el].

[8] B. Douçot, L. B. Ioffe, and J. Vidal, Phys. Rev. B69, 214501 (2004), arXiv:quant-ph/0302104v2.

[9] Zh.-Y. Xue, Z. D. Wang, and Sh.-L. Zhu, Phys. Rev. A77, 024301 (2008), arXiv:0710.2407v4 [quant-ph].

[10] B. Paredes and I. Bloch, Phys. Rev. A77, 023603 (2008), arXiv:0711.3796v1 [cond-mat.other].

[11] L. M. Duan, E. Demler, and M. D. Lukin, Phys. Rev. Lett. 91
090402 (2003), arXiv: cond-mat/0210564v2.

[12] A. Micheli, G. K. Brennen, and P. Zoller, Nat. Phys. 2, 341 (2006), arXiv: quant-ph/0512222v2.

[13] L. Jiang et al., Nature Phys. 4, 482 (2008), arXiv: $0711.1365 \mathrm{v} 1$ [quant-ph].

[14] Y. J. Han, R. Raussendorf, and L.-M. Duan, Phys. Rev. Lett. 98 (2007) 150404, arXiv: quant-ph/0702031v1.

[15] I. Bloch, J. Dalibard, and W. Zwerger, Rev. Mod. Phys. 80, 885 (2008), arXiv:0704.3011v2 [cond-mat.other].

[16] A. Yu. Kitaev, Annals Phys. 321, 2 (2006), arXiv : cond-mat/ 0506438v3 [cond-mat.mes-hall].

[17] Ch. Zhang et al., Proc. Natl. Acad. Sci. USA 10418415 (2007), arXiv:quant-ph/0609101v4.

[18] S. Dusuel, K. P. Schmidt, and J. Vidal, Phys. Rev. Lett. 100, 177204 (2008), arXiv:0802.0379v1 [cond-mat.other].

[19] Using auxiliary particles as "moving heads" was already proposed in J. I. Cirac and P. Zoller, Nature 404, 579 (2000); K. G. H. Vollbrecht, E. Solano, and J. I. Cirac, Phys. Rev. Lett. 93, 220502 (2004), arXiv: quant-ph/0405014v2; T. Calarco et al., Phys. Rev. A70, 012306 (2004), arXiv: quant-ph/0403197v1.

[20] M. Anderlini et al., Nature 448, 452 (2007), arXiv: 0708.2073v1 [quant-ph].

[21] S. Kuhr et al., Phys. Rev. Lett. 91, 213002 (2003), arXiv: quant-ph/0304081v1.

[22] M. Greiner et al., Nature 415, 39 (2002).

[23] D. Bacon, Phys. Rev. A78, 042324 (2008), arXiv: 0806.2160v2 [quant-ph].

[24] D. Jaksch et al., Phys. Rev. Lett. 81, 3108 (1998), arXiv: cond-mat/9805329v3.

[25] E. Dennis et al., J. Math. Phys. 43, 4452 (2002), arXiv:quant-ph/0110143v1.

[26] C. Mochon, Phys. Rev. A69, 032306 (2004), arXiv: quant-ph/0306063v2.

[27] D. Jaksch et al., Phys. Rev. Lett. 82, 1975 (1999), arXiv: quant-ph/9810087v1.

[28] G. K. Brennen et al., Phys. Rev. Lett. 82, 1060 (1999), arXiv:quant-ph/9806021v4.

[29] M. D. Lukin et al., Phys. Rev. Lett. 87, 037901 (2001), arXiv: quant-ph/0011028v1.

[30] F. Verstraete et al., Phys. Rev. Lett. 96, 220601 (2006), arXiv:quant-ph/0601075v2.

[31] G. K. Brennen, M. Aguado, and J. I. Cirac, arXiv: $0901.1345 \mathrm{v} 1$ [quant-ph].

[32] S. Bravyi, M. B. Hastings, and F. Verstraete, Phys. Rev. Lett. 97, 050401 (2006), arXiv: quant-ph/0603121v1.

[33] E. Charron et al., Phys. Rev. A74, 012308 (2006), arXiv: quant-ph/0603138v1.

[34] D. Schrader et al., Phys. Rev. Lett. 93, 150501 (2004), arXiv: quant-ph/0409037v1.

[35] R. Stock, E. L. Bolda, and I. H. Deutsch, Phys. Rev. Lett. 91, 183201 (2003), arXiv: quant-ph/0304093v2.

[36] F. W. Strauch et al., Phys. Rev. A77, 050304(R) (2008), arXiv:0712.1856v1 [quant-ph].

[37] S. S. Bullock and G. K. Brennen, J. Phys. A: Math. Theor. 40, 3481 (2007), arXiv:quant-ph/0609070v2.

[38] M. de Wild Propitius, Topological interactions in broken gauge theories, Ph. D. thesis, University of Amsterdam, 1995, arXiv: hep-th/9511195v1.

[39] M. Levin and X.-G. Wen, Phys. Rev. B67, 245316 (2003), arXiv: cond-mat/0302460v2 [cond-mat.str-el].

[40] J. Cho, Phys. Rev. Lett. 99, 020502 (2007), arXiv : quant-ph/ $0703185 \mathrm{v} 2$.

[41] A. V. Gorshkov et al., Phys. Rev. Lett. 100, 093005 (2008), 
arXiv:0706.3879v2 [quant-ph]. 